\title{
A Statistical Model for Combustion Resonance from a DI Diesel Engine with Applications
}

\author{
Timothy Bodisco ${ }^{a *}$, Samantha Low $\mathrm{Choy}^{a}$, Assaad Masri ${ }^{b}$, Richard J. \\ Brown $^{a}$ \\ ${ }^{a}$ Science and Engineering Faculty, Queensland University of Technology, Australia \\ ${ }^{b}$ Faculty of Engineering and Information Technologies, University of Sydney, Australia
}

\begin{abstract}
Introduced in this paper is a Bayesian model for isolating the resonant frequency from combustion chamber resonance. The model shown in this paper focused on characterising the initial rise in the resonant frequency to investigate the rise of in-cylinder bulk temperature associated with combustion. By resolving the model parameters, it is possible to determine: the start of pre-mixed combustion, the start of diffusion combustion, the initial resonant frequency, the resonant frequency as a function of crank angle, the in-cylinder bulk temperature as a function of crank angle and the trapped mass as a function of crank angle. The Bayesian method allows for individual cycles to be examined without cycle-averaging - allowing inter-cycle variability studies. Results are shown for a turbo-charged, common-rail compression ignition engine run at $2000 \mathrm{rpm}$ and full load.
\end{abstract}

Keywords: Combustion Resonance, Resonant Frequency, Bayesian Modelling, Markov-chain Monte Carlo, In-cylinder Pressure Analysis

\section{Introduction}

Typically researchers interested in frequency content from engine signals (in-cylinder pressure, vibration and acoustic emission) have employed fast Fourier transforms (FFT) $[1,2]$. However, many authors have used more advanced techniques that are able to capture non-stationary frequencies, such

\footnotetext{
${ }^{*}$ Corresponding Author.

e-mail: timothy.bodisco@qut.edu.au

Preprint submitted to Mechanical Systems and Signal Processing

December 3, 2013
} 
as: finite element analysis [3], Wigner-Ville methods [4-6], Hilbert transforms $[5,7]$ and continuous wavelet transforms $[8,9]$. Fast Fourier transforms are common practice in basic spectral analysis mostly because of their ease of use and computational efficiency $[10,11]$. However, many assumptions are made when using them - for example, the assumption of periodic stationary frequencies. Moreover, they can also have low resolution and are sensitive to noise and incomplete data $[12,13]$ - low resolution may make it difficult to resolve close together frequencies [14]. In his pioneering 1988 work, Bretthorst [12] explains that if there is complex phenomena, or evidence of more than a single stationary harmonic frequency, the Fourier method may yield incorrect or misleading results.

In order to avoid any misinterpretation of the data, the method of data analysis should be carefully selected. Under discussion here is the use of Bayesian modelling with the application of isolating features of the combustion resonance in a heavy-duty Cummins, multi-cylinder, turbo-charged, common-rail, direct-injection diesel engine. Of specific interest is the spectral content contained in the in-cylinder pressure signal; in particular the resonant frequency that occurs as a result of combustion. Isolation of this frequency is important as it is related to the speed of sound and hence in-cylinder bulk temperature $[1-3,5,15,16]$.

The challenge with isolating the resonant frequency information from the combustion resonance is that the frequency itself is an evolving frequency. In order for a FFT to produce meaningful results, multiple periods of data are required [17] - it is counter-intuitive in an application where a frequency is evolving to simultaneously analyse multiple periods of data. An immediate implication of this is that the FFT method will not be able to capture the transient nature of this signal, even if the data is analysed in windows such as those found in a spectrogram - the results would only be able to indicate a trend and likely not provide any definitive information. For a detailed study of combustion resonance, with the goal of isolating the initial resonant frequency (at the onset of combustion) and characterising its rise as the combustion chamber temperature increases, the FFT method will not be satisfactory.

Continuous wavelet transforms (CWT) have emerged in recent years as a solution to the instantaneous frequency problem and have been used in a wide range of applications $[18,19]$. In comparison to the spectrogram method (using short-time Fourier transforms), the time window is not held constant [18] - it is advantageous to not have constant time windows, automated or 
even manual selection of windows may cut useful information [20]. Rather, the time window of each wavelet is varied to fit the data; therefore, there is always a trade-off between the frequency and time resolutions. In relation to the application described in this paper, resolving a single evolving frequency with respect to crank-angle, the CWT method has a few key short-comings. Namely: the results of the CWT are typically presented in scales which need to be estimated as frequencies (causing frequency resolution issues), difficulty with accurate automated feature detection (the results are normally analysed visually, other algorithms would need to be carefully generated to automate the feature extraction), wavelet selection effects the results and whilst the CWT method does isolate evolving frequencies well, there is no indication of uncertainty in the result and resolution issues can make detailed tracking of the frequency of interest difficult.

Other advanced techniques, which are capable of capturing the transient nature of engine signals, have issues of their own. These issues generally relate to undesirable assumptions inherent with the analysis technique, susceptibility to noise, resolution and cross-talk. Statistical modelling, within the Bayesian paradigm, is suggested to overcome these issues. As this is not a one-size-fits-all method, using this method is at the expense of simplicity [16]. However, it can be argued that in data analysis more emphasis should be given to scientific interest and less to mathematical convenience [21]. Here a solution is provided to this problem that does require access to computational support.

In the Bayesian paradigm all assumptions must be explicitly stated; this allows the analyst more control compared to other techniques [22]. Whilst this adds complexity, it also ensures that the analyst is completely aware of the problem they are solving and reduces the potential risk of obtaining a misleading result. Moreover, in this application it allows for the analysis to be conducted independently, on individual engine cycles; thereby, allowing for inter-cycle variability studies and effectively removing the need for ad hoc methods such as cycle averaging [16, 23].

Some work in this area has already been conducted by Bodisco et al. $[16,23]$. Much like the current work detailed in this paper, previous work has resolved parameters in an empirical form of the in-cylinder pressure signal. In the original work [16] model development was shown for isolating the resonant frequency as a function of time in a four-cylinder DI naturally aspirated diesel engine - the model implementation was performed in prepackaged software, WinBUGS [24]. However, the final model was kept sim- 
ple and made some assumptions to reduce complexity. One such assumption was that the resonant frequency only decayed - i.e. there was no initial rise in frequency with the corresponding increase in in-cylinder bulk temperature with combustion. In that work [16] the final conceptual model was:

$$
\begin{aligned}
y & =s(t) \sim \mathrm{N}(\mu(t), \tau) \\
\mu(t) & =f_{1}(t)+H(t-\delta) \sum_{i=2}^{3} f_{i}(t) \\
f_{i}(t) & =A_{i} e^{-\lambda_{i} t} \sin \left(W_{i} \omega_{0} e^{-a_{i} t} t+\phi\right)
\end{aligned}
$$

where $s(t)$ is the in-cylinder pressure signal with the motoring frequency information removed and is Normally distributed with time-varying mean, $\mu(t)$, and precision, $\tau$. The time-varying mean consists of the summation of three frequency $\left(\omega_{i}(t)=W_{i} \omega_{0} e^{-a_{i} t}\right)$ and amplitude $\left(A_{i}(t)=A_{i} e^{-\lambda_{i} t}\right)$ decaying sinusoidal waves with the same phase shift, $\phi$, where $i$ identifies the sinusoidal. In this model, sinusoidals $i=\{2,3\}$ are zero until $t>\delta$ as controlled by the step function $H(t-\delta)$.

Extending the previous work [16], this paper will demonstrate the use of a more sophisticated model to estimate the rise of the resonant frequency in a heavy-duty Cummins, multi-cylinder, turbo-charged, common-rail, directinjection diesel engine. Accurate isolation of the resonant frequency allows for an in-depth investigation into the combustion temperature. Moreover, as the Bayesian method allows for each cycle to be analysed independently, the inter-cycle variability of this important parameter can be investigated.

Experimental investigation into the combustion bulk temperature using an advanced technique, such as described in this paper, could significantly aid in the analysis of alternative fuels. In diesel engines, emission formation, especially $\mathrm{NO}_{x}$, is temperature dependent $[25,26]$. The strong dependency between emission and temperature indicates the need for more advanced methods of investigation into in-cylinder bulk temperature and temperature rate rise.

\section{Experimental Configuration}

Experiments were conducted at the QUT Biofuel Engine Research Facility (BERF) in August 2012. The engine under investigation is a $5.9 \ell$ in-line six-cylinder, turbo-charged, common-rail Cummins diesel engine. This is a 
heavy-duty Euro 3 engine with advanced injection timing (around top dead centre) and variable injection pressure (250-1800 bar). Table 1 contains the technical specifications of the engine and data acquisition equipment.

Data was collected at $200 \mathrm{kHz}$ use a Data Translation (DT9832) analogueto-digital converter and National Instruments LabView. This engine was equipped with a high resolution crank angle sensors (operated at 0.5 crank angle degree resolution) and the data was collected asynchronously. The location of the pressure transducer can be seen in Figure 1, an elevation is not shown because the cylinder head is flat.. For the experimental results shown, the engine was run at $2000 \mathrm{rpm}$ on neat automotive diesel at full load $(760 \mathrm{Nm})$. A more detailed description of the engine setup can be found in Ref. [27].

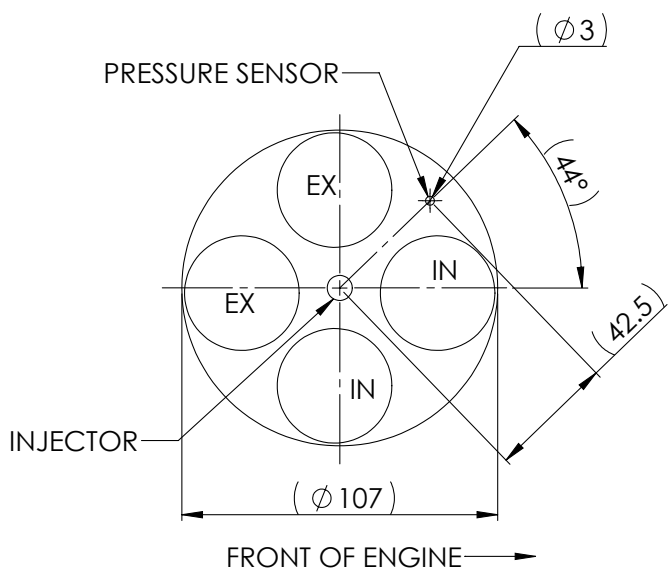

Figure 1: Location of in-cylinder pressure transducer

\section{Experimental Data}

Using a two channel analogue Krohn-Hite model 3202 filter, the in-cylinder pressure signal was band-pass filtered. The first channel was a high-pass filter with a threshold of $4 \mathrm{kHz}$ and the second a low-pass filter with a threshold of $12 \mathrm{kHz}$. In contrast to the ignition delay study [23], this frequency band 
Table 1: Engine and data acquisition specifications

\begin{tabular}{|ll|}
\hline Engine Specifications & \\
\hline Make & Cummins ISBe220 31 \\
Capacity & $5.9 \ell$ \\
Maximum power & $162 \mathrm{~kW}$ at $2000 \mathrm{rpm}$ \\
Maximum torque & $820 \mathrm{Nm}$ at $1500 \mathrm{rpm}$ \\
Number of cylinders & 6 \\
Number of valves per cylinder & 4 \\
Compression ratio & $17.3: 1$ \\
Bore & $102 \mathrm{~mm}$ \\
Stroke length & $120 \mathrm{~mm}$ \\
Dynamometer & Electronically controlled water \\
& brake dynamometer \\
Injection system & Common-rail \\
\hline Data acquisition & \\
\hline Pressure transducer & Kistler piezoelectric transducer \\
& (6053CC60) \\
Analogue-to-digital converter & Data Translation (DT9832) \\
Software & National Instruments LabView \\
Sample rate & 200 kHz \\
Data collected & In-cylinder pressure \\
& Band-pass filtered in-cylinder pres- \\
& sure (allowing 4-12 kHz) \\
& Diesel injection timing \\
& Crank-angle rotation information \\
\hline
\end{tabular}


was kept very close to the frequencies of interest. This was done to ensure that noise and vibration not directly related to the combustion resonance was minimised. A pilot study was performed with low-pass threshold frequencies ranging from $8-20 \mathrm{kHz}$. It was determined that this study could be performed at any of these filter settings; however, the band-pass range of $4-12 \mathrm{kHz}$ was chosen because a low-pass threshold at $12 \mathrm{kHz}$ produces a signal free from high frequency noise that qualitatively looks identical to those of lower thresholds.

Using the same acquisition system as described [23, 27], the band-pass filtering took place whilst the signal was still in its analogue form; therefore, the dependent scale in Figure 2 is in Volts. Isolating the resonant frequency from in-cylinder pressure is scale independent; hence, there is no need to convert the input signal from a voltage signal into a pressure signal. Figure 2 shows an example of the band-pass filtered signal - it is taken from the same cycle as the in-cylinder pressure trace shown in Figure 3. The rise in signal ( $\sim 362$ degrees crank angle) immediately prior to the start of the combustion resonance ( $\sim 354$ degrees crank angle) evident in Figure 2 is attributable to electronic noise from the injection signal and is not part of the combustion resonance.

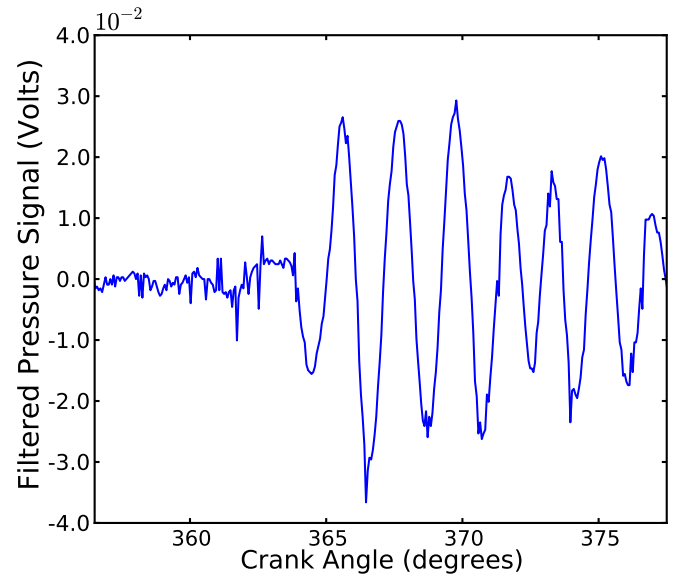

Figure 2: Band-pass (4-12 kHz) filtered pressure signal, full load, $2000 \mathrm{rpm}$ 


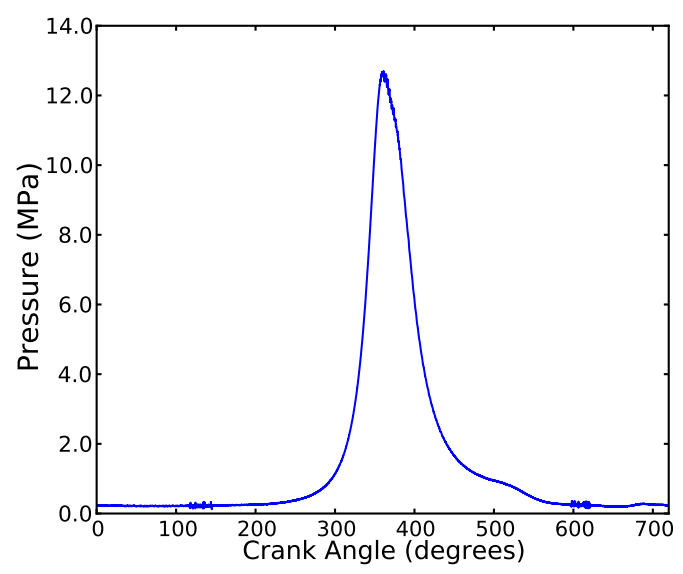

Figure 3: Pressure vs crank angle plot, full load, $2000 \mathrm{rpm}$

\section{Conceptual Model}

In Ref. [23], a statistical model was used to determine the start of combustion from in-cylinder pressure. The model employed was:

$$
\begin{aligned}
y & =s(t) \sim \mathrm{N}\left(\mu(t), \sigma_{y}\right) \\
\mu(t) & =H(t-\delta) A \sin \left(\frac{2 \pi}{\lambda} \omega t+\phi\right),
\end{aligned}
$$

where, $s(t)$, the band-pass in-cylinder pressure signal (such as the example in Figure 2), is assumed to be Normally distributed about some time varying mean, $\mu(t)$, with some standard deviation, $\sigma_{y}$ - the independent variable $t$ refers to the discrete time representation in the data related to the sampling rate $(200,000 \mathrm{~Hz})$. The change point factor, $H(t-\delta)$, switches the signal from Gaussian noise about 0 to a periodic sine wave of frequency $\omega-\lambda$ is the sample-rate in $\mathrm{Hz}$ and $\phi$ is the phase-shift. Therefore, resolving the change point parameter, $\delta$, resolves the start of combustion.

As the work in Ref. [23] was primarily interested in resolving the start of combustion only, the window this analysis took place in was restricted to 200 data points. This allowed for the assumption that the resonance was from a stationary frequency. However, the present work is interested in characterising the rise in the resonant frequency and accurately resolving the initial resonant frequency. 
This can be achieved with only slight modification to the model shown in Equation 1. In this implementation the amplitude, $A$, will be predetermined as a function of time and the resonant frequency, $\omega$, will be resolved as a function of time:

$$
\begin{aligned}
y & =s(t) \sim \mathrm{N}\left(\mu(t), \sigma_{y}\right) \\
\mu(t) & =H\left(t-\delta_{1}\right) A(t) \sin \left(\frac{2 \pi}{\lambda} \omega(t) t+\phi\right),
\end{aligned}
$$

The amplitude, henceforth termed the frequency envelope, is predetermined to ensure the frequency is correctly resolved - the other model parameters are conditional on the amplitude. This approach helped improve model fit, and is a generalisation of a common Bayesian computational practice to exploit hierarchial structure where possible. Hence, minimising issues with incorrect frequency envelope estimates artificially reducing the data likelihood estimates. Moreover, having the frequency envelope as a model input saves computation time, by not having to resolve it as part of the modelling process.

The method for resolving the frequency envelope used in this work has been kept simple, as it is not necessary for it to be precise. First, meanaverage smoothing was applied to the band-pass filtered in-cylinder signal to reduce the effects of noise. This smoothed signal was then differentiated and the timing of all of the turning points was identified (when the differentiated signal $=0$ ). The absolute value of the original band-pass filtered in-cylinder signal (before smoothing) at each of the identified turning points was found and the frequency envelope was then determined by linearly interpolating between those points. This method is computationally efficient and consistently yielded a result that adequately fit the frequency envelope.

For modelling the rise of the resonant frequency, a multiplicative model was chosen as it can rise slowly or quickly and the rate only relies on one parameter - multiplicative models can be directly related to exponential models but are computationally easier to work with. Heat release has been modelled previously using an Arrhenius type exponential equation [28, 29]. In an auto-ignition process, such as that found in diesel engines, a pool of radicals needs to be developed as the fuel is gradually heating and mixing with the surrounding oxygen and combustion products. This may occur in a region just downstream of the exit plane of the penetrating spray jet where sufficient vaporisation has taken place. Ignition kernels will form and these would be 
convected with the flow until they encounter combustible mixtures, which are then ignited leading to extensive heat release. It is notable that in spray jets auto-igniting in a hot vitiated co-flow, the heat release is minimal in the region where the auto-igniting kernels are developed and increases significantly downstream in the jet where the main combustion is taking place [30, 31]. Such a scenario is also expected in the diesel engine considered here. Based on this, it is expected that the resonant frequency will rise slowly at the start of pre-mixed combustion (when the auto-igniting kernels are developing) and then more rapidly at the start of diffusion combustion (characterised by extensive heat release), eventually followed by a slow decline. The model used to empirically represent the resonant frequency in this study is:

$$
\omega(t)= \begin{cases}\beta_{0} & t<\delta_{1} \\ \beta_{1} \omega(t-1) & \delta_{1} \leq t<\delta_{2} \\ \beta_{2} \omega(t-1) & \delta_{2} \leq t<\delta_{3} \\ \beta_{3} \omega(t-1) & t \geq \delta_{3} .\end{cases}
$$

As the empirical relationship given in Equation 3 is a multiplicative model, $\omega(t-1)$ refers to the value of $\omega$ at the previous data point. The initial resonant frequency is given by $\beta_{0}$, the initial rise in resonant frequency (from pre-mixed combustion) is characterised by $\beta_{1}$, the rapid rise in resonant frequency (from diffusion combustion) by $\beta_{2}$ and the decline in the resonant frequency by $\beta_{3}$. Similarly, the model parameter $\delta_{1}$ (in both Equations 2 and 3) corresponds to the start of pre-mixed combustion, $\delta_{2}$ corresponds to the start of diffusion combustion and $\delta_{3}$ corresponds to the timing at which the resonant frequency begins to decline.

The Bayesian approach will provide marginal posterior plausibilities of each of the parameter values, based on the observed data. This allows the estimation of the model parameters. Bayes theorem states that the posterior distribution is proportional to the product of the likelihood of the data given the parameters and the priori distribution of the parameters [32].

$$
p(\theta \mid y) \propto p(y \mid \theta) p(\theta),
$$

for this case, $\theta=\left\{\delta_{1}, \delta_{2}, \delta_{3}, \beta_{0}, \beta_{1}, \beta_{2}, \beta_{3}, \phi \sigma_{y}\right\}$ and $y_{i}=s\left(t_{i}\right), t_{i} \in \mathcal{T}$.

\section{Priors}

In a Bayesian approach, parameter values require specification of prior plausibility. In this analysis, what is known about each model parameter is 
independent of the other parameters; therefore:

$$
p(\theta)=p\left(\delta_{1}\right) p\left(\delta_{2}\right) p\left(\delta_{3}\right) p\left(\beta_{0}\right) p\left(\beta_{1}\right) p\left(\beta_{2}\right) p\left(\beta_{3}\right) p(\phi) p\left(\sigma_{y}\right) .
$$

The prior distributions should encompass all plausible values a parameter can take [32].

Using the same logic shown in Ref. [23], $\phi \sim \operatorname{Unif}(0, \pi)$ and $\beta_{0} \sim$ Unif(5000, 7000). Combustion is assumed to occur approximately 4.5 degrees after the start of injection (approximately 100 data points after the nominal start of injection) [23]; therefore, $\delta_{1} \sim \mathrm{N}(100,25)$. This prior assumes a $95 \%$ probability that combustion will occur in the time period, $50 \leq t \leq 150$.

The apparent rate of heat release diagram (generated from cycle-averaged data), shown in Figure 4, is constructed using the first law of thermodynamics as described by Heywood [33] and does not account for heat losses. Examination of Figure 4 reveals an inflection at approximately 370 degrees (approx 250 data points after the nominal start of injection). This position corresponds to the timing at which the temperature begins to increase more rapidly. The same prior given to $\delta_{1}$ is assigned to $\delta_{2}, \delta_{2} \sim \mathrm{N}(250,25)$. Experience and observation of the data, informs the last change point parameter, $\delta_{3}$. A slight decrease in the resonant frequency is typically observed shortly after the rapid rise; therefore, $\delta_{3} \sim \mathrm{N}(300,25)$. Additionally, $\delta_{3}$ has the restriction that it muct ho mroatar than $\delta$.

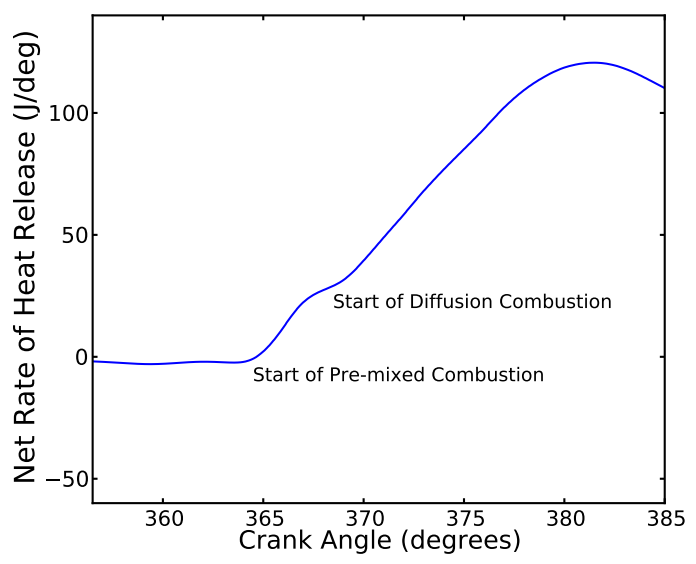

Figure 4: Apparent net rate of heat release, full load, $2000 \mathrm{rpm}$

Of interest in this study is the resonant frequency and how it evolves as a function of time. Because this is a multiplicative model, the parameter 
values of $\beta_{1}, \beta_{2}$ and $\beta_{3}$ will be near 1. The model shown in Ref. [23] worked because the start of the combustion resonance can be assumed to be somewhat stationary. Therefore, $\beta_{1}$ was assigned a prior that was very close to $1, \beta_{1} \sim \mathrm{N}(1.00005,0.00005)$. If a starting frequency of approximately 5700 $\mathrm{Hz}$ is assumed and a peak frequency of $6500 \mathrm{~Hz}$, an approximation of $\beta_{2}$ of 1.002 can be shown. Therefore, $\beta_{2} \sim \mathrm{N}(1.002,0.001)$. Both $\beta_{1}$ and $\beta_{2}$ were restricted to be greater than 1 .

Knowledge of $\beta_{3}$ is more difficult to predict. Unlike $\beta_{1}$ and $\beta_{2}$, where it can be assumed they are greater $1, \beta_{3}$ could potentially represent the beginning of the decline in the resonant frequency or it could represent the slowing down of the evolution. The prior selected has assumed a decline, but has not enforced it, $\beta_{3} \sim \mathrm{N}(0.9999,0.001)$.

The model parameter $\sigma_{y}$ is there to fit the Gaussian noise in the data. In Bayesian modelling it is common to consider the precision $\left(\tau=\frac{1}{\sigma_{y}^{2}}\right)$, rather than the standard deviation [32]. In a state of ignorance, this parameter is often assigned an uninformative Gamma prior, $\tau \sim \operatorname{Gamma}(0.01,0.01)$.

\section{Model Implementation}

Estimation of the posterior distributions of the model parameters is performed using a standard Bayesian computational approach based on simulation. The Metropolis-Hastings algorithm, a special case of the Markov-chain Monte Carlo (MCMC) algorithm [32], has been used in this analysis as described in Ref. [23] - this section is a summary from the more detailed explanation in Ref. [23]. In this implementation candidate parameters, $\theta^{*}$, are sampled from a proposal distribution dependent on the current parameter value, $\theta^{*} \sim \mathrm{R}(\theta)$. For the parameters, $\left\{\beta_{0}, \beta_{1}, \beta_{2}, \beta_{3}, \phi \sigma_{y}\right\}$ :

$$
\theta^{*} \sim \mathrm{N}\left(\theta^{m-1}, \sigma_{\theta}\right)
$$

and for the parameters, $\left\{\delta_{1}, \delta_{2}, \delta_{3}\right\}$ :

$$
\theta^{*} \sim \operatorname{Unif}\left(\theta^{m-1}-n, \theta^{m-1}+n\right)
$$

where, $\sigma_{\theta}$ and $n$ are tuning parameters which determine the potential range of the 'walk' at each iteration and $m=1, \ldots, M$ denotes the $m^{\text {th }}$ MCMC simulation from the chain. Therefore,

$$
\theta^{m}= \begin{cases}\theta^{*} & \text { with probability } \alpha \\ \theta^{m-1} & \text { otherwise. }\end{cases}
$$


where,

$$
\alpha=\min \left\{1, \frac{R\left(\theta^{*}\right)}{R\left(\theta^{m-1}\right)} \frac{p\left(\theta^{*} \mid \cdot\right)}{p\left(\theta^{m-1} \mid \cdot\right)}\right\}
$$

and - denotes the full set of parameters omitting the parameter of interest, here generically denoted as $\theta$.

The results from the model had a tendency to not correctly resolve the start of combustion, $\delta_{1}$. This issue occurred because of the predetermined frequency envelope causing issues with convergence. Potential solutions to this issue included: modelling the frequency envelope as part of the model instead of having it as an input parameter, developing a hierarchical model or predetermining the start of combustion and having it as an input parameter. Using the model described in Ref. [23] to determine the start of combustion, $\delta_{1}$, and then using $\delta_{1}$ as an input parameter (non-updatable) was determined as the most computational efficient and reliable solution. Moreover, using the predetermined frequency envelope ensures that once the frequency has converged to the correct solution that the model will fit the data well and is therefore an important input parameter and removing it could result in convergence issues and greatly increased computation time.

\section{Simulation Results}

Figure 5 shows the model fit to the signal shown in Figure 2. It can be seen by observation, or by the residual plot shown in Figure 6, that the model has fit the data very well - indicating accurate isolation of the resonant frequency. This observation is made because the residual plot shows no distinctive features (i.e. it appears to be noise only without any repeating features). Table 2 shows the mean and standard deviation for each model parameters prior and posterior distributions. 
Table 2: Model parameter prior and posterior distributions

\begin{tabular}{|c|c|c|c|c|}
\hline \multicolumn{3}{|c|}{ Prior } & \multicolumn{2}{|l|}{ Posterior } \\
\hline Parameter & Mean & $\begin{array}{l}\text { Standard } \\
\text { Deviation }\end{array}$ & Mean & $\begin{array}{l}\text { Standard } \\
\text { Deviation }\end{array}$ \\
\hline$\beta_{1}$ & 1.00005 & 0.00005 & 1.00023 & $3.204 \times 10^{-5}$ \\
\hline$\beta_{2}$ & 1.002 & 0.001 & 1.00101 & $2.869 \times 10^{-5}$ \\
\hline$\beta_{3}$ & 1.9999 & 0.001 & 1.00004 & $3.381 \times 10^{-5}$ \\
\hline$\delta_{1}$ & $\begin{array}{c}100 \\
(362.3 \text { degrees })\end{array}$ & $\begin{array}{c}25 \\
\text { (1.5 degrees) }\end{array}$ & $\begin{array}{c}122 \\
\text { (363.7 degrees) }\end{array}$ & $\begin{array}{c}4 \\
\text { (0.3 degrees) }\end{array}$ \\
\hline$\delta_{2}$ & $\begin{array}{c}250 \\
(371.5 \text { degrees })\end{array}$ & $\begin{array}{c}25 \\
\text { (1.5 degrees) }\end{array}$ & $\begin{array}{c}242 \\
(371.0 \text { degrees })\end{array}$ & $\begin{array}{c}2 \\
(0.2 \text { degrees })\end{array}$ \\
\hline$\delta_{3}$ & $\begin{array}{c}300 \\
(374.6 \text { degrees })\end{array}$ & $\begin{array}{c}25 \\
\text { (1.5 degrees) }\end{array}$ & $\begin{array}{c}297 \\
(374.5 \text { degrees })\end{array}$ & $\begin{array}{c}1 \\
(0.1 \text { degrees })\end{array}$ \\
\hline & $\begin{array}{l}\text { Lower } \\
\text { Bound }\end{array}$ & $\begin{array}{l}\text { Upper } \\
\text { Bound }\end{array}$ & Mean & $\begin{array}{l}\text { Standard } \\
\text { Deviation }\end{array}$ \\
\hline $\begin{array}{c}\beta_{0} \\
\phi\end{array}$ & $\begin{array}{c}5000 \\
0\end{array}$ & $\begin{array}{c}7000 \\
\pi\end{array}$ & $\begin{array}{l}5502 \\
0.27\end{array}$ & $\begin{array}{c}43 \\
0.18\end{array}$ \\
\hline
\end{tabular}

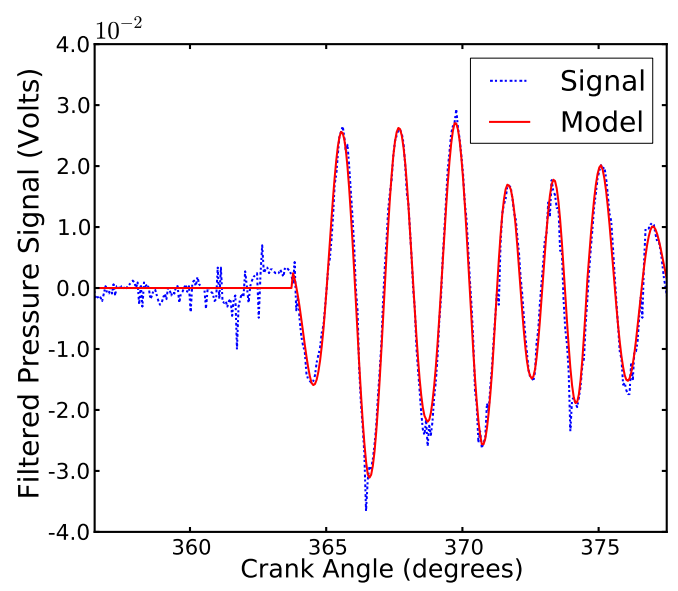

Figure 5: Model fit to signal shown in Figure 2 


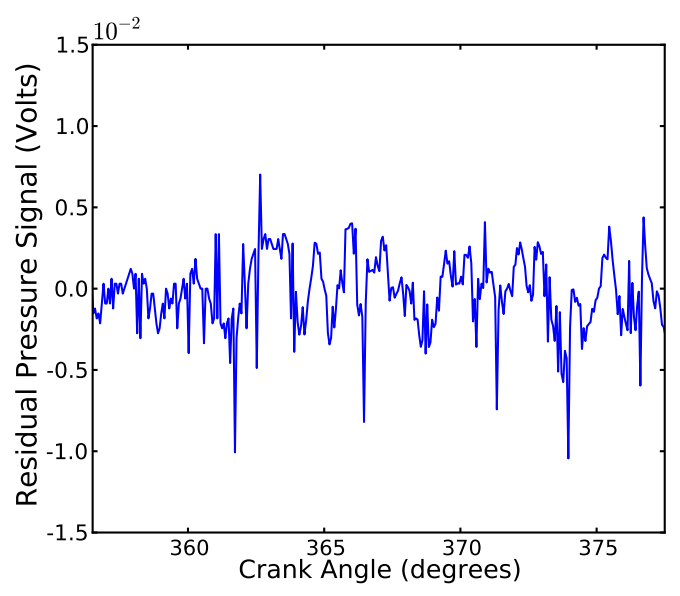

Figure 6: Residual plot showing that subtraction of the fitted model from the signal shown in Figures 2 and 5

Hence, as a function of crank angle, the resonant frequency of the signal shown in Figure 2, is modelled in Figure 7. For comparison to the continuous wavelet transform method, Figure 8 shows the corresponding result from a continuous wavelet analysis using 256 scales. It can be seen that both methods have isolated the frequency information well; however, owing to the resolution in the Bayesian method, results in Figure 7 allow for the determination of the start of pre-mixed and diffusion combustion and a greater ability to track the evolution of the frequency as a function of crank angle (or time). Some key features, however, can be noted in Figure 8 that correspond to Figure 7. Namely: the general range of the frequency is similar, the start of pre-mixed combustion can be seen at approximately 364 degrees and a sharper rise in frequency is also evident at approximately 371 degrees. 


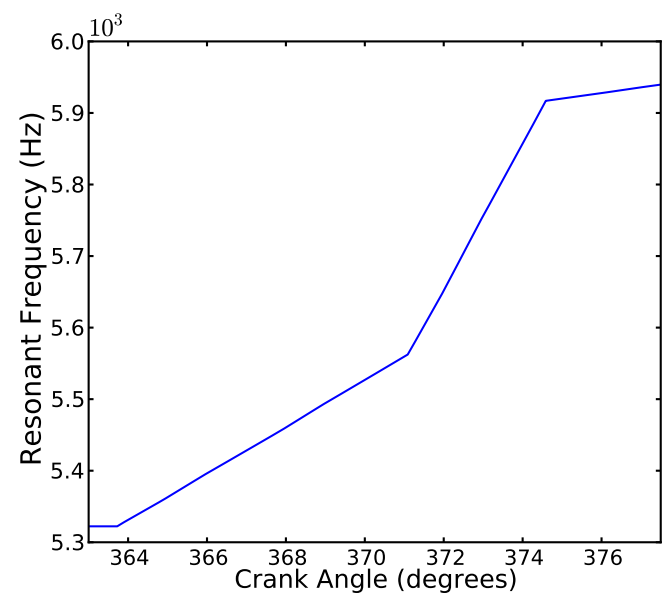

Figure 7: Example resonant frequency with respect to crank angle from model fit shown in Figure 5

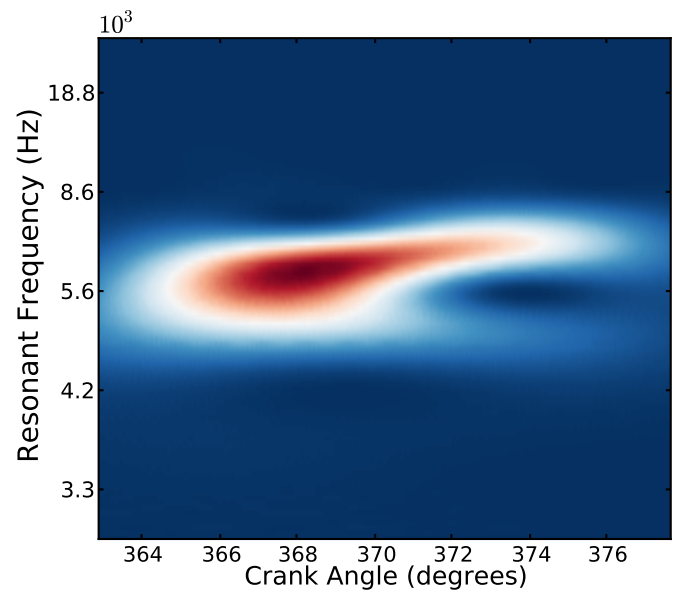

Figure 8: Resonant frequency of the signal shown in Figure 2 isolated using a continuous wavelet transform

From 500 consecutive engine cycles, Figure 9 shows the distribution of the initial resonant frequency, $\beta_{0}$. 


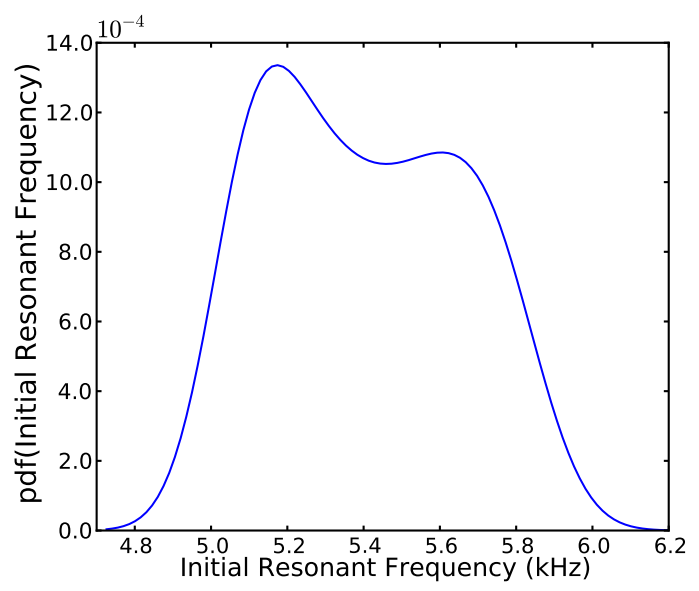

Figure 9: Distribution of the initial resonant frequency over 500 consecutive cycles

Although determined as an input parameter from the start of combustion model [23], Figures 10 and 11 show the distribution of the start of pre-mixed combustion and the corresponding ignition delay.

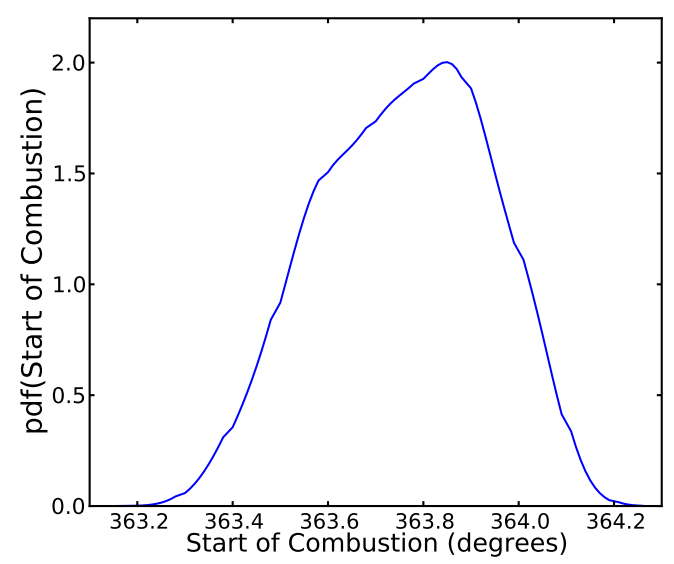

Figure 10: Distribution of the start of pre-mixed combustion over 500 consecutive cycles 


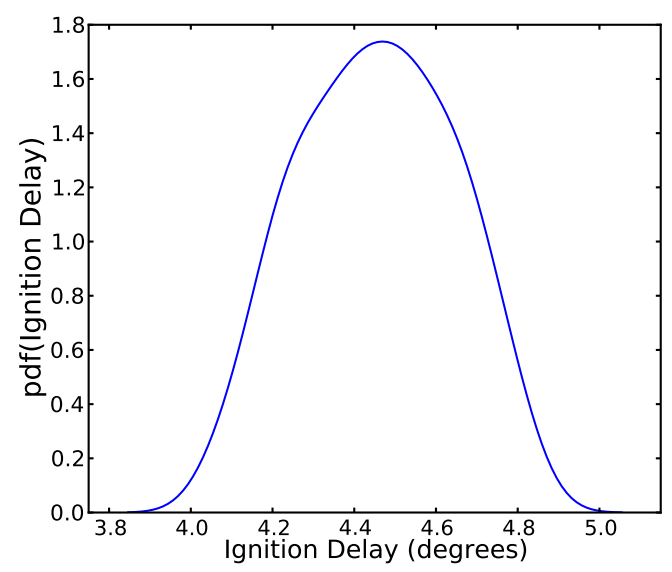

Figure 11: Distribution of the ignition delay over 500 consecutive cycles

The model parameter $\delta_{2}$ isolates the start of diffusion combustion - characterised by an increase in the rate of temperature rise. Figure 12 shows the distribution of the start of diffusion combustion. This timing matches well with an inflection seen in the net rate of heat release, shown in Figure 4.

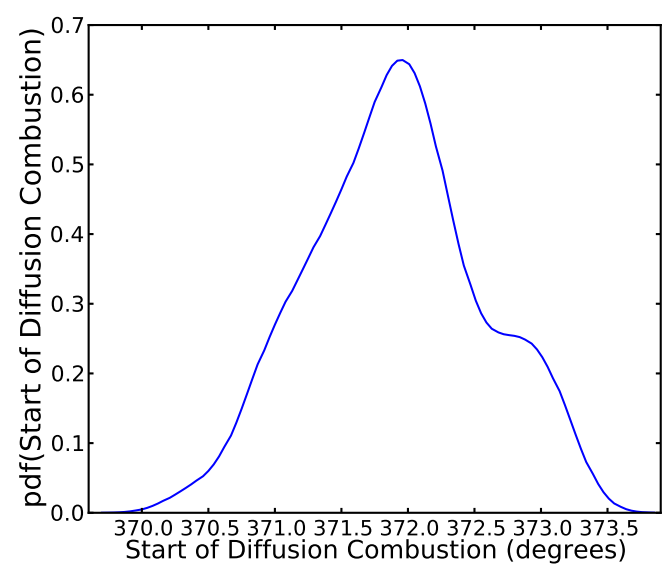

Figure 12: Distribution of the start of diffusion combustion over 500 consecutive cycles

Corresponding to these change point parameters, Figure 13 shows the distribution of the multiplicative parameter $\beta_{1}$ which corresponds to the initial 
rise in temperature during pre-mixed combustion and Figure 13 shows distribution of the multiplicative parameter $\beta_{2}$ which corresponds to the more rapid rise in temperature from diffusion combustion.

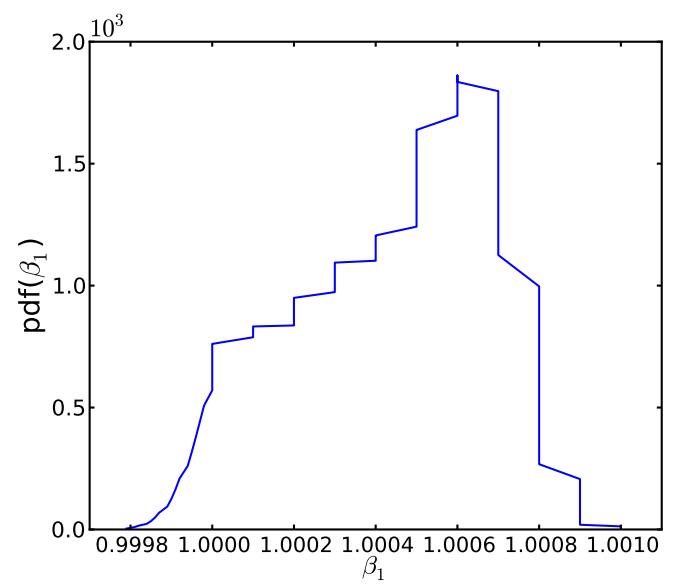

Figure 13: Distribution of the initial multiplicative parameter, $\beta_{1}$, over 500 consecutive cycles

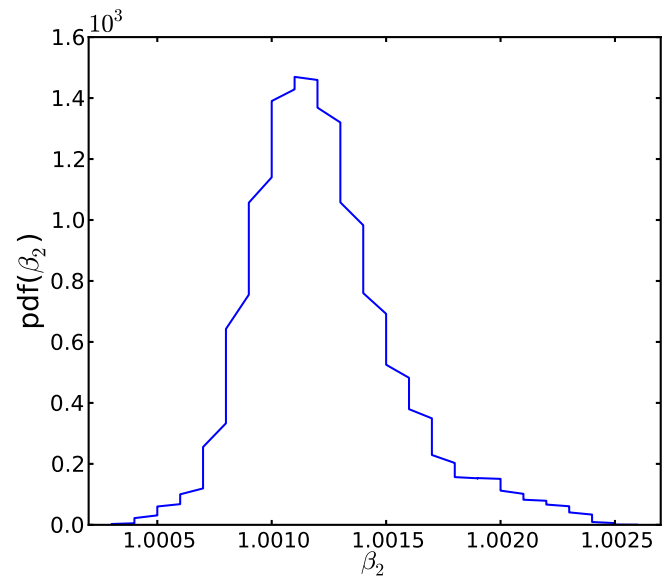

Figure 14: Distribution of the diffusion combustion multiplicative parameter, $\beta_{2}$, over 500 consecutive cycles

The results for the multiplicative parameter $\beta_{3}$ were slightly different to expected. When the model was developed it was assumed that at the end of 
the diffusion combustion period the in-cylinder bulk temperature (and hence resonant frequency) would start decreasing. However, the results shown in Figure 15 indicated that this has only shown to be true on some cycles. On the majority of cycles, Figure 15 indicates that the rise in resonant frequency is slowed or perhaps stationary for a period before it decreases.

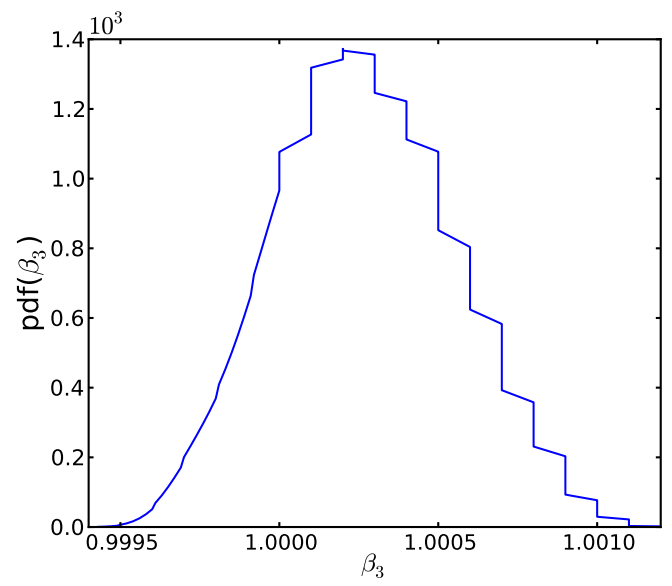

Figure 15: Distribution of the reduction in combustion intensity parameter, $\beta_{3}$, over 500 consecutive cycles

\section{Application}

A key feature of using a Bayesian modelling technique is being able to analyse individual cycles. Therefore, the analyst is able to investigate the inter-cycle variability of not only the model parameters, as shown in Section 7 , but also the inter-cycle variability of the operating characteristics derived from the model results. Potential applications for these results are determining the in-cylinder bulk temperature and the trapped mass as a function of time $[3,16]$.

A nominal in-cylinder bulk temperature can be determined from the relationship to the speed of sound by assuming ideal behaviour and uniform gas composition in the combustion chamber-whilst these are not true assumptions, it does allow for an interesting investigation into the bulk temperature in the combustion chamber. The speed of sound, $c$, is related to the resonant frequency, $f$, by the following relationship [3]:

$$
c=\frac{f B}{\alpha_{n m}}
$$


where, $B$ is the cylinder bore and $\alpha_{n m}$ is a non-dimensional value associated with the frequency mode - in this study the resonant frequency represents the first circumferential frequency; therefore, $\alpha_{n m}=0.5861[3,16]$. The speed of sound is also related to temperature [3]:

$$
c^{2}=\gamma R T
$$

where, $\gamma$ is the ratio of specific heats, $R$ is the characteristic gas constant and $T$ is the in-cylinder bulk temperature. Combining Equations 4 and 5:

$$
T=\frac{1}{\gamma R}\left(\frac{f B}{\alpha_{n m}}\right)^{2}
$$

and relating Equation 6 to the ideal gas law allows the determination of the trapped mass, $m$, as a function of time:

$$
m(t)=\gamma P(t) V(t)\left(\frac{\alpha_{n m}}{f(t) B}\right)^{2}
$$

Owing to potential imperfections in the combustion chamber walls and design features that deviate from a perfectly round profile, the in-cylinder bulk temperature and therefore the trapped mass determined from Equations 6 and 7, respectively, will not be correct [34]. However, a correction factor can be determined from knowledge of the trapped mass in the cylinder at the onset of combustion. In the engine setup utilised in this experiment in-take air and fuel flows are measured. The nominal distribution of trapped mass (at the onset of combustion), determined from Equation 7, is shown in Figure 16. Under the assumption that at the start of combustion there has been negligible blow-by, a correction factor can be found.

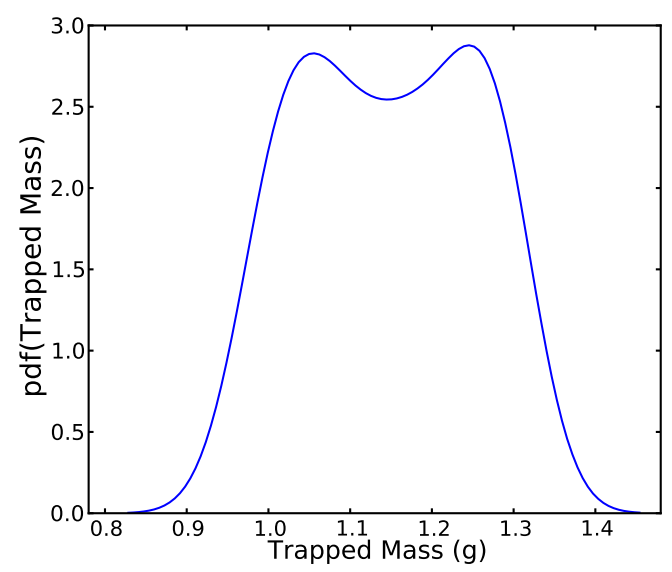


Figure 16: Distribution of the cycle resolved nominal trapped mass over 500 consecutive cycles

A rearrangement of the ideal gas law (with the correction factor $(\zeta=1.074$ ), shown in Equation 8) allows an in-cylinder bulk temperature profile to be created for each individual engine cycle, example in Figure 17, and investigation into the inter-cycle variability of the in-cylinder bulk temperature at the start of combustion, shown in Figure 18. This in-cylinder bulk temperature is associated with the spatial average in-cylinder temperature. In reality, it is expected that the changes between the different phases of combustion would be smoother; however, Figure 17 does give an accurate indication of the spatial average in-cylinder temperature with respect to crank angle.

The bi-modal distribution of values shown in Figure 18 is interesting. It is showing that the predominate initial in-cylinder temperature is approximately $2000 \mathrm{~K}$; but, it is also showing a significant second mode at approximately $2400 \mathrm{~K}$. This bi-modal distribution is likely caused by instabilities in the control of the dynamometer. Data collected from this engine setup often shows a periodic time dependency, the spread of the distribution shown in Figure 18 is indicating that this variability has a significant impact on the in-cylinder temperature immediately prior to combustion. A bi-modal distribution, rather than a uni-modal distribution, is likely caused by bias in the sampling period - the dynamometer instability generally fluctuates with a period of approximately 50 seconds; therefore, a 60 second sample (as shown in this paper) will likely give bias to the repeated section of the sample period.

$$
T=\frac{P V}{\zeta m R}
$$




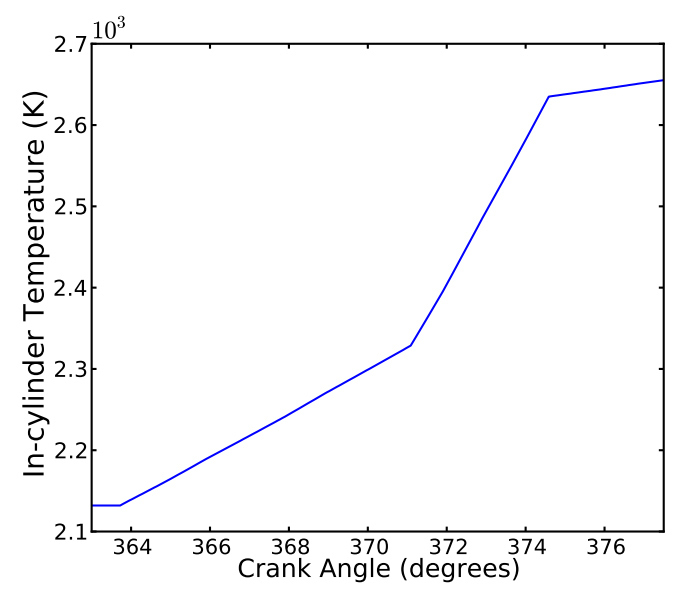

Figure 17: In-cylinder bulk temperature as a function of crank angle

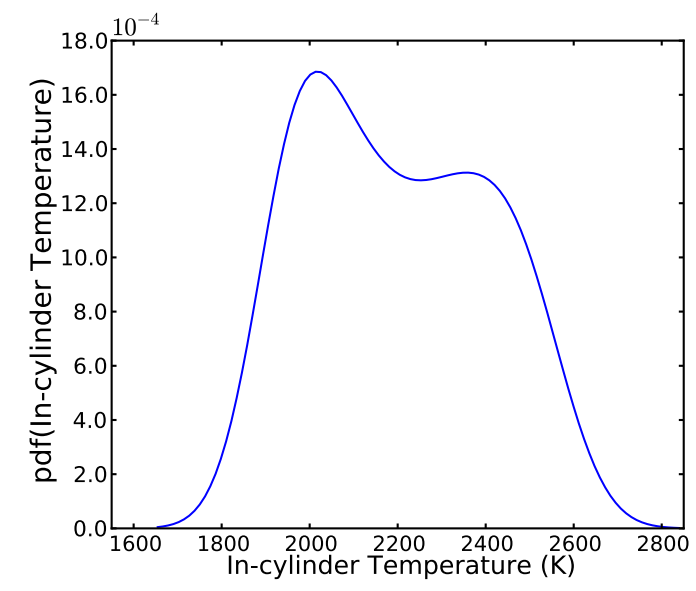

Figure 18: Distribution of the initial in-cylinder bulk temperature across 500 consecutive cycles

\section{Conclusion}

This paper has introduced a Bayesian model for analysing the combustion chamber resonant frequency. The model employed in this paper focused on capturing the rise in the resonant frequency associated with the increase 
in in-cylinder temperature from combustion. Applied to a band-pass filtered in-cylinder pressure signal, from a compression ignition engine, this model is capable of directly determining: the start of combustion, the start of diffusion combustion, the initial resonant frequency and the resonant frequency as a function of time. It has also been demonstrated that knowledge of the resonant frequency allows further investigation into the in-cylinder bulk temperature and trapped mass as a function of time. Results from the Bayesian model have been shown for 500 consecutive cycles of a turbocharged, common-rail injection $5.9 l$ diesel engine run at full load and 2000 rpm.

The application of this technique to a modern compression ignition engine has shown that it is able to resolve a crank angle dependent (or time dependent) average spatial in-cylinder temperature profile and is useful for investigating the inter-cycle variability of the engine. A bi-modal distribution of in-cylinder temperature was shown and explained to be caused by engine operation instability. Moreover, this method for investigating the combustion chamber resonance also yields a new metric for describing the rate of increase in temperature during combustion, in terms of the model multiplicative parameters, $\beta_{i}$. Applications for this can include a more rigorous examination of the effect of alternative fuels and fuelling strategies, allowing the analyst a greater breadth of information to draw conclusions from.

\section{Acknowledgements}

The authors wish to thank Mr. Noel Hartnett for his work in maintaining the engine for research. Further thanks also to Technologist Mr. Ken

McIvor for his assistance in setting up the data acquisition software and to Mr. Daniel Naylor for providing the technical drawing in Figure 1. For technical advice relating to thermoacoustics, the authors would also like to acknowledge Professor Wolfgang Polifke of Technische Universität München. This work was undertaken under an Australian Research Council Linkage Grant (LP0775178) in association with Peak3 P/L.

\section{References}

[1] A. J. Torregrosa, A. Broatch, X. Margot, Combustion chamber resonances in direct injection automotive diesel engines: a numerical approach, International Journal of Engine Research 5 (1) (2004) 83-91. 
[2] F. Payri, A. Broatch, B. Tormos, V. Marant, New methodology for incylinder pressure analysis in direct injection diesel engines: application to combustion noise, Measurement Science and Technology 16 (2) (2005) $540-547$.

[3] R. Hickling, D. A. Feldmaier, F. H. K. Chen, J. S. Morel, Cavity resonances in engine combustion chambers and some applications, Acoustical Society of America Journal 73 (1983) 1170-1178.

[4] L. Stankovic, J. F. Bohme, Time-frequency analysis of multiple resonances in combustion engine signals, Signal Processing 79 (1) (1999) $15-28$.

[5] Y. Ren, R. B. Randall, B. E. Milton, Influence of the resonant frequency on the control of knock in diesel engines, Proceedings of the Institution of Mechanical Engineers: Part D: Journal of Automobile Engineering. London 213 (2) (1999) 127-133.

[6] C. S. Bhat, P. H. Meckl, J. S. Bolton, J. Abraham, Influence of fuel injection parameters on combustion-induced noise in a small diesel engine, International Journal of Engine Research 13 (2) (2012) 130-146.

[7] H. Li, Z. Zhang, Diesel engine condition classification based on mechanical dynamics and time-frequency image processing, Dynamical Systems (2010) 397-408.

[8] W. Li, F. Gu, A. D. Ball, A. Y. T. Leung, C. E. Phipps, A study of the noise from diesel engines using the independent component analysis, Mechanical Systems and Signal Processing 15 (6) (2001) 1165-1184.

[9] H. Jun, H. Bing, Identification of diesel front sound source based on continuous wavelet transform, in: The 18th International Congress on Acoustics, Kyoto, Japan, 2004.

[10] P. Duhamel, M. Vetterli, Fast Fourier transforms: a tutorial review and a state of the art, Signal Processing 19 (4) (1990) 259-299.

[11] N. E. Huang, Z. Shen, S. R. Long, M. C. Wu, H. H. Shih, Q. Zheng, N. Yen, C. C. Tung, H. H. Liu, The empirical mode decomposition and the Hilbert spectrum for nonlinear and non-stationary time series 
analysis, Proceedings: Mathematical, Physical and Engineering Sciences 454 (1998) 903-995.

[12] G. L. Bretthorst, Excerpts from Bayesian spectrum analysis and parameter estimation, Maximum Entropy and Bayesian Methods in Science and Engineering 1 (1988) 75-145.

[13] E. T. Jaynes, Bayesian spectrum and chirp analysis, Maximum-Entropy and Bayesian Spectral Analysis and Estimation Problems (1987).

[14] L. Dou, R. Hodgson, Bayesian inference and Gibbs sampling in spectral analysis and parameter estimation: I, Inverse Problems 11 (5) (1995) 1069-1085.

[15] J. F. Bohme, D. Konig, Statistical processing of car engine signals for combustion diagnosis, in: IEEE Seventh SP Workshop on Statistical Signal and Array Processing, 1994, pp. 369-374.

[16] T. Bodisco, R. Reeves, R. Situ, R. J. Brown, Bayesian models for the determination of resonant frequencies in a DI diesel engine, Mechanical Systems and Signal Processing 26 (2012) 305-314.

[17] P. C. Gregory, T. J. Loredo, A new method for the detection of a periodic signal of unknown shape and period, Astrophysical Journal 398 (1) (1992) 146-168.

[18] Z. K. Peng, F. L. Chu, Application of the wavelet transform in machine condition monitoring and fault diagnostics: a review with bibliography, Mechanical Systems and Signal Processing 18 (2) (2004) 199-221.

[19] A. K. Sen, R. Longwic, G. Litak, K. Gorski, Analysis of cycle-to-cycle pressure oscillations in a diesel engine, Mechanical Systems and Signal Processing 22 (2) (2008) 362-373.

[20] V. Poggi, D. Faeh, D. Giardini, Time-frequency-wavenumber analysis of surface waves using the continuous wavelet transform, Pure and Applied Geophysics 170 (3) (2012) 319-335.

[21] G. Box, G. Tiao, Bayesian Inference in Statistical Analysis, John Wiley and Sons, 1992. 
[22] G. L. Bretthorst, C. R. Smith, Bayesian analysis of signals from closelyspaced objects, in: Infrared Systems and Components III, Proc. SPIE 1050, 1989.

[23] T. Bodisco, S. Low Choy, R. J. Brown, A Bayesian approach to the determination of ignition delay, Applied Thermal Engineering 60 (1-2) (2013) 79-87.

[24] D. J. Spiegelhalter, A. Thomas, N. G. Best, WinBUGS Version 1.2 User Manual, MRC Biostatistics Unit (1999).

[25] A. S. Ramadhas, C. Muraleedharan, S. Jayaraj, Performance and emission evaluation of a diesel engine fuelled with methyl esters of rubber seed oil, Renewable Energy 30 (12) (2005) 1789-1800.

[26] A. Frassoldati, T. Faravelli, E. Ranzi, A wide range modeling study of $\mathrm{NO}_{x}$ formation and nitrogen chemisty in hydrogen combustion, International Journal of Hydrogen Energy 31 (15) (2006) 2310-2328.

[27] T. Bodisco, R. J. Brown, Inter-cycle variability of in-cylinder pressure parameters in an ethanol fumigated common rail diesel engine, Energy 52 (1) (2013) 55-65.

[28] K. Nishida, H. Hiroyasu, Simplified three-dimensional modeling of mixture formation and combustion in a D.I. diesel engine, SAE Paper 890269 (1989).

[29] D. Jung, D. Assanis, Multi-zone DI diesel spray combustion model for cycle simulation studies of engine performance and emission, SAE Paper 2001-01-1246 (2001).

[30] W. O'Loughlin, A. Masri, A new burner for studying auto-ignition in turbulent dilute sprays, Combustion and Flame 158 (2011) 1577-1590.

[31] W. O'Loughlin, A. Masri, The structure of the auto-ignition region of turbulent dilute methanol sprays issuing in a vitiated co-flow, Flow Turbulence Combustion 89 (2012) 13-35.

[32] A. Gelman, J. B. Carlin, H. S. Stern, D. B. Rubin, Bayesian Data Analysis: Second Edition, Chapman \& Hall/CRC, 2003. 
[33] J. B. Heywood, Internal Combustion Engine Fundamentals, McGrawHill, Inc., 1988.

[34] R. B. Randall, Frequency Analysis, Brueel \& Kjaer, 1987. 